\title{
Analysis and Simulation of Wired VoIP Networks compared to Wireless VoIP
}

\author{
Eliud M. Manyinsa ${ }^{1}$, S.Musyoki ${ }^{2}$, H. Tarus ${ }^{3}$ \\ ${ }^{I}$ Telecommunication and Information Engineering, At Jomo Kenyatta University of Agriculture and Technology \\ (P.O. Box 27129-00100 Nairobi Kenya) \\ ${ }^{2}$ School of Electrical and Electronics Engineering, At Technical University of Kenya (P.O. Box 52428-00200 \\ Nairobi Kenya) \\ ${ }^{3}$ West Indian Ocean Cable Company Limited (P.O. Box 14137-00800 Nairobi Kenya)
}

\begin{abstract}
Voices over Internet Protocol (VoIP) allow users to make calls and receive calls by connecting through internet protocols to deliver voice data. The reasons behind growing popularity of VoIP include; low cost, efficient bandwidth and flexibility over Public Switched Telephone Network (PSTN). In this study we present a VoIP system using Local Area Network (LAN) and Wireless Local Area Network (WLAN) for connectivity. These systems will be referred to as VoWLAN and Wired VoIP.VoWLAN offers a significantly extended operational range. Users increasingly rely on WLAN connections creating a challenge due to increase in demand. The designed model used in this paper is simulated to derive measurements of selected parameters such as delay, jitter, packet loss and throughput. The performance of voice communication over wired connections to its WLAN counterpart is done using NS-3 simulator. With interest in WLAN as the main wireless internet connectivity, VoIP becomes a central topic for voice communication over WLAN network due to less cost as compared to other mode of communication. The paper evaluates the performance difference between wired and wireless VoIP networks. It has noted that the wireless nodes are affected by its proximity to the Access Points, acting as the receiver. In close proximity, the wireless nodes will perform better than the wired network. However, the latency and jitter is unstable for wireless networks, especially as the distance increases between the mobile nodes and the Access Points.
\end{abstract}

Keywords: VoIP, WLAN, VoWLAN, NS-3, UDP, TCP

\section{Introduction}

VoIP operates by transferring voice signals between IP addresses, which means that those signals have to transform into pieces of data small enough to transmit. Vocals samples from the sender are broken down into voice "packets", which are given routing information and sent to the receiving end. The packets transmit oneby-one, then re-form as close to the original state as possible, creating one whole voice. This process compresses the voice signal, and then decompresses the signal for the receiver. This paper is for better understanding of how VoIP works in underlying layers of the network. The wired VoIP and VoWLAN topologies are built by using NS3. In order to make the simulation more realistic like in the real world, the background traffic is added. To have better understanding of the difference between the VoIP on wired and wireless network, packet loss, throughput, delay and jitter are measured and analysed.

\section{History of VoIP}

\section{Background Information}

Voice over Internet Protocol (VoIP) is a communication protocol which runs over Internet Protocol (IP) networks. In Aug 1974, Network Voice Protocol (NVP) was first tested over Arpanet. The first VoIP application, Speak Freely, was released to public in 1991. Three years later, a free VoIP application for Linux, MTALK, appeared [1]. After forty years of developments, VoIP has become a very mature communication protocols. VoIP was implemented in MSN in 2005. Google Voice service permitted VoIP connections through Gmail or Google Talk in 2009. The most recently use of VoIP is Facebook Inc. It launched free calling app for IPhone in January 2013.

\section{Transport Layer Protocol}

TCP and UDP are two main transport layer protocols designed for end-to-end communication between two applications and implements some control functions.

\section{Voice over TCP}

Transmission Control Protocol (TCP) is a transport layer protocol.TCP provides a connection oriented, reliable, byte stream service and uses a 32-bit end-to-end checksum. The term connection-orientedmeans the 
two applications in this case voice using TCP must establish a TCP connection with each other before they canexchange data. It is a full duplex protocol, meaning that each TCP connection supports a pair of bytestreams, one flowing in each direction.TCP includes a flow-control mechanism for each of these bytestreams that allow the receiver to limit how much data the sender can transmit.

\section{Voice over UDP}

The User Datagram Protocol (UDP) is a simple transmission without doing any error checks. However, the advantage of UDP is less delay. Theoretically, packet loss must be within certain range in order to deliver normal condition voice package.Time-sensitive applications often use UDP because dropping packets is preferable to waiting for delayed packets, which may not be an option in a real-time system. Communication is achieved by transmitting information in onedirection from source to destination without verifying the readiness or state of the receiver.However, oneprimary benefit of UDP over TCP is the application to voice over internet protocol (VoIP) where anyhandshaking would hinder clear voice communication [2].

Table1: Comparison between UDP and TCP

\begin{tabular}{|l|l|}
\hline TCP & UDP \\
\hline $\begin{array}{l}\text { Reliable-It manages message acknowledgement, retransmission } \\
\text { and timeout }\end{array}$ & $\begin{array}{l}\text { Unreliable- There is no concept of acknowledgement, } \\
\text { retransmission and timeout }\end{array}$ \\
\hline $\begin{array}{l}\text { Ordered-The first message reaches the receiving application } \\
\text { first }\end{array}$ & $\begin{array}{l}\text { Not Ordered-if two messages are sent to the recipient, the order in } \\
\text { which they arrive cannot be predicted. }\end{array}$ \\
\hline $\begin{array}{l}\text { Heavyweight-It requires three packets to set up a socket } \\
\text { connection, before any data is sent. }\end{array}$ & $\begin{array}{l}\text { Lightweight-There is no ordering of messages, no tracking } \\
\text { connections. It is a small transport layer designed on top of IP. }\end{array}$ \\
\hline $\begin{array}{l}\text { Streaming-Data is read as a byte stream, no distinguishing } \\
\text { indications are transmitted to signal message (segment) } \\
\text { boundaries. }\end{array}$ & $\begin{array}{l}\text { Datagram-Packets are sent individually and are checked for } \\
\text { integrity only if they arrive. Packets have definite boundaries } \\
\text { which are honored upon receipt. }\end{array}$ \\
\hline $\begin{array}{l}\text { Congestion Control-It implements the congestion control } \\
\text { mechanism. }\end{array}$ & $\begin{array}{l}\text { No congestion Control-It does not avoid congestion and it is } \\
\text { possible for high bandwidth application to trigger congestion } \\
\text { collapse, unless they implement congestion control measures at } \\
\text { the application level }\end{array}$ \\
\hline
\end{tabular}

\section{LAN Vs WLAN}

LAN stands for Local Area Network, which is a collective of network devices in a certain location that are connected together by switches and/or routers that facilitate communication of network elements hence a wired network. WLAN stands for wireless LAN, where the data is transmitted over the air through the use of wireless transmitters and receivers.Below is the comparison between Wired LAN and WLAN

Table 2. Comparison between LAN and WLAN

\begin{tabular}{|l|l|}
\hline LAN & WLAN \\
\hline LAN devices are based on IEEE802.3 standards & WLAN devices are based on IEEE802.11 family of standards \\
\hline LAN devices use electric signals to transmit the data & $\begin{array}{l}\text { WLAN devices use high energy radio frequency waves to transmit } \\
\text { the data }\end{array}$ \\
\hline $\begin{array}{l}\text { Electric signals flow through the cables. Hence wired } \\
\text { connection is needed between devices which are connected to } \\
\text { the LAN }\end{array}$ & $\begin{array}{l}\text { Radio frequency waves travel in the space, a physical connection } \\
\text { is not needed between devices which are connected to the WLANs }\end{array}$ \\
\hline LAN supports full duplex mechanism for communication. & WLAN uses half duplex mechanism for communication \\
\hline $\begin{array}{l}\text { LANs suffer less interference as electric signals travel using } \\
\text { ables }\end{array}$ & $\begin{array}{l}\text { WLAN suffers from interference of various types during travel } \\
\text { from source to the destination }\end{array}$ \\
\hline LANs use CSMA/CD to detect collisions in the network & WLANs use CSMA/CA to avoid collisions in the network. \\
\hline
\end{tabular}

\section{Implementation, Simulations, and Calculations Simulation with ns-3}

The tests will be carried out in NS-3. C++ scripts will be used to parse the resulting simulation trace files, and evaluate the throughput, latency, jitter, and packet loss. The test topology includes two VoIP clients, sending two-way traffic to each other to simulate normal voice communication. The VoIP traffic is running at the start of simulation. At a later time, background traffic isbeing added to evaluate its effects on the VoIP traffic. The background traffic is sent via separated nodes/clients, while only sharing the transmission paths. Two protocols will be used for each of the wired and wireless scenarios: UDP and TCP. Both protocols are available in NS-3 as source agents [3]. 


\section{Wired VoIP System Topology}

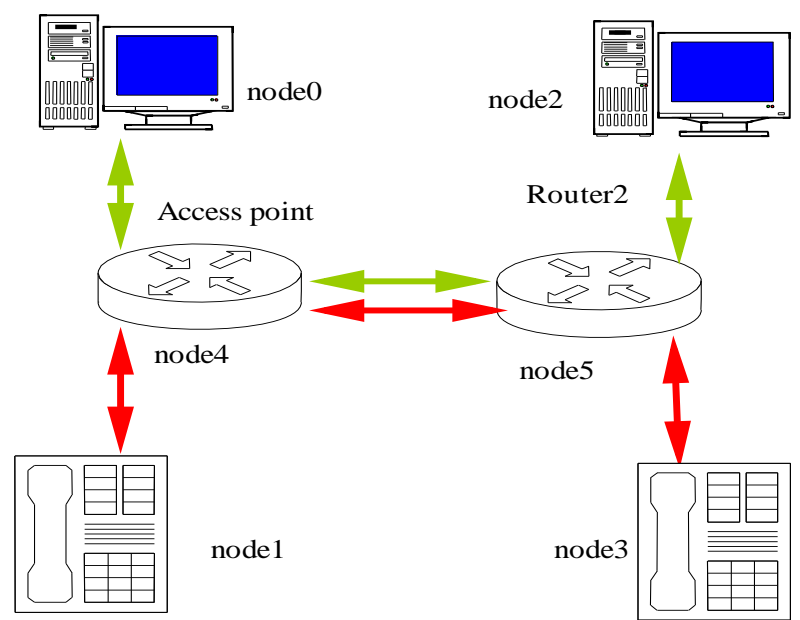

Figure 1: Wired VoIPsimulation

In implementation, G.711 codec is used since it is a commonly used audio codec. This codec uses a 64kbps bit rate and packet size of 160 bytes [4]. The background traffic is sending at a constant bit rate of 128kbps. The topology for the wired setup is shown in Figure 1, with red denoting two-way traffic between the VoIP clients. The green denotes two-way traffic between the background sources. Node 1 \& Node 3is a VoIP traffic and Node $0 \&$ Node 2is the background. There is two-way traffic for both background and VoIP. The bandwidth limits for the routers (Node 4 \& Node 5) is set to the same bitrate as the background traffic. Once the background traffic starts, the links between the routers is overloaded and the impacton the VoIP application can be seen.The queue build-up exists at the centre link, serving as a bandwidth bottleneck.

\section{VoWLAN System Topology}

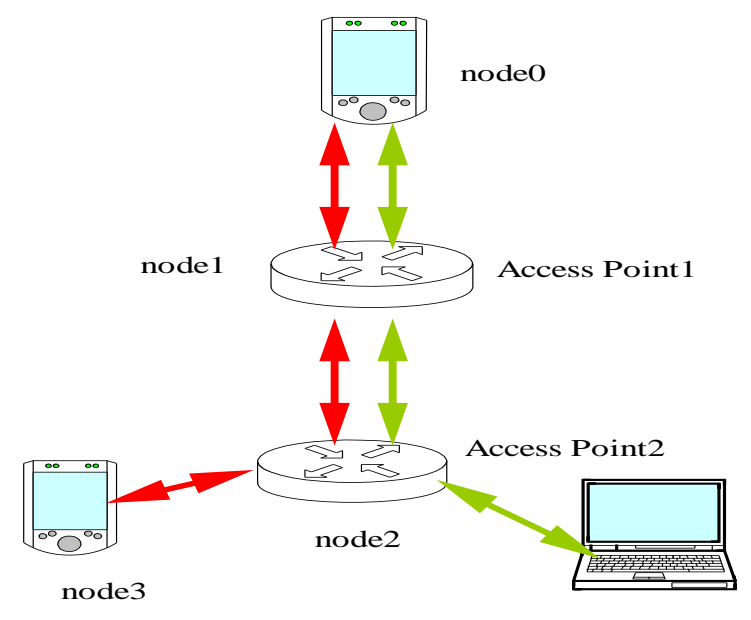

node4

Figure 2: VoWLANSimulation

The wireless test setup is similar to the wired topology, with the same traffic route shown in figure 2. Mobile node 3 is sending VoIP traffic (160 bytes with 0.020 s interval) to node 0 . The traffic arrives at the Access Point node, travels through node 1 and is received by the client at node 0 . Node 0 sends VoIP traffic via the same route back to mobile node 3. This will ensure the performances between WLAN and wired tests can be accurately compared.The Access Point 2 acts as a receiver for wireless traffic from nodes 3 and 4. The bandwidth bottleneck for the wireless setup is the duplex link between node 1 (Access Point1) and the Access Point2, which will generate dropped packets upon traffic exceeding the transmission speed of 64kbps. 


\section{Throughput}

\section{Performance Calculations}

Throughput is the average rate of successful data pass over a communication channel. It is measured in bytes/sec. For example, from client Node 0 to client Node 1 , both clients connect to server Node A and Node B, the throughput of VoIP refers to the total amount of voice data transfer between Node 0 and Node 1 . The average throughput will produce a single value showing the average throughput for the entire duration of the simulation. The formula is as following:

Average Throughput $=($ Total number of bytes receive in destination node $) /$ Simulation Time-------(1)

\section{Packet Loss}

Packet loss happens when packets of data travelling across a computer network never reaches its destination. It occurs when packets enter a queue when it is full. This is for the case of Drop-Tail queuing method, which will drop the last packet attempting to enter the queue upon reaching queue limit. The cumulative packet loss tells the total numbers of packets dropped throughout the simulation.

Cumulative Packet Loss $=($ Total number of Packets Dropped $) /$ Simulation runtime

The cumulative packet loss can be plotted on a graph progressively over each second, and will also give summed total of packet loss at the end of the runtime. This will allow easy comparison between various scenarios and protocols.

\section{Latency}

Latency is a measure of time delay experienced in a system. In simulation, the delay is measured by taking the time difference between when a packet is sent from the source node, and when it reaches its destination. The latency (end-to-end delay) will be measured for each packet sent from a VoIP client, and reaches its partner. The instantaneous latency formula is given as:

Instantaneous Latency $=$ Receive time of destination node - Send time of source node-------(3)

The instantaneous latency can be presented graphically, where the end-to-end delay (in seconds, y-axis) for a specific packet is posted at the time it was received by the destination node ( $\mathrm{x}$-axis). The Average Latency is a single value, calculated from the formula given below:

Average Latency = cumulative total of instantaneous latency / simulation runtime-----(4)

The average latency produces a single value which can be easily used to carry out performance comparisons between different scenarios.

\section{Packet Delay Variation (Jitter)}

Jitter is an informal name for IP packet delay variation (IPDV), but it is often used in electronics and telecommunication. Jitter is the undesired deviation from true periodicity of an assumed periodic signal in computer network. "As an example, say packets are transmitted every $20 \mathrm{~ms}$. If the $2 \mathrm{nd}$ packet is received $30 \mathrm{~ms}$ after the $1 \mathrm{st}$ packet, IPDV $=-10 \mathrm{~ms}$. This is referred to as dispersion. If the $2 \mathrm{nd}$ packet is received $10 \mathrm{~ms}$ after the 1 st packet, IPDV $=+10 \mathrm{~ms}$. This is referred to as clumping [5]."

Instantaneous Jitter $=$ Current Latency - Previous Latency--------(5)

Instantaneous Jitter is graphed over the simulation period, and each data point will be mapped to its respective time in the Instantaneous Latency graph. This allows examination of the stability of the network as the traffic pattern constantly changes throughout the simulation.

The average jitter can be calculated as:

Instantaneous Jitter $=$ sum $($ Current Latency - Previous Latency $) /$ simulation runtime-------(6) 


\section{Results and Discussion}

The performance between wired and WLAN VoIP networks is compared for UDP and TCP protocols. The background traffic initiates at 2 seconds into the simulation.

UDP - Throughput

Throughput-Wired VoIPvs VoWLAN

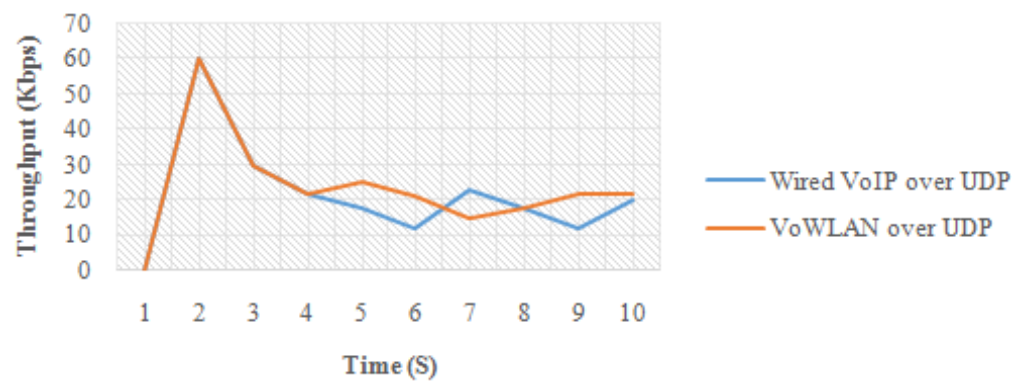

Figure 3: Throughput - Wired VoIP VS VoWLAN

The resulting throughput between wired and wireless VoIP networks under UDP is similar. This is due to the mobile nodes being placed relatively close to the Access Points, so that the signal is strong enough to mirror a wired connection. As seen from the figures, without any background traffic, both VoWLAN and wired networks easily reach the throughput of 60kbps, guaranteeing reliable voice traffic between the clients.

\section{UDP - Packet Lost}

\section{Packet Lost-Wired VoIP vs VoWLAN}

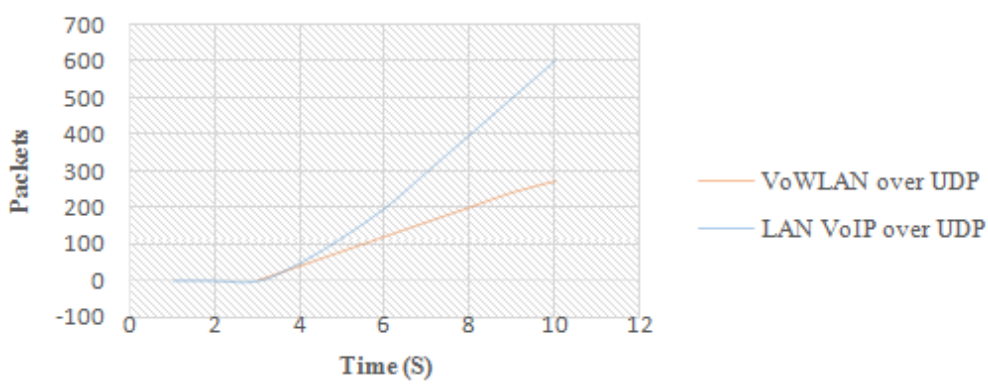

Figure4: Packet Lost - Wired VoIP VS VoWLAN

The packet loss is significantly more for wired than for wireless under the UDP protocol. This does not match the theoretical results, in which the instability of wireless packet transfers will result in higher packet loss. This result is due to the queuing type of the duplex links, as there are three separate queues between sender and receiver, which results in undesired queue stacking characteristics resulting in significant amount of packet loss over long periods of times.

\section{UDP - Latency}

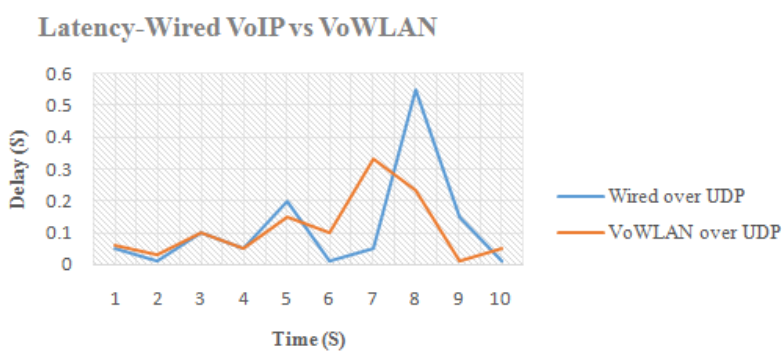

Figure5: Latency - Wired VoIP VS VoWLAN 
The latency is shorter for the wireless network than the wired network under the UDP protocol. This is due to the mobile nodes being in too close proximity of the Access Points, which resulted in better performance for the wireless signals than for the pre-set delays existing in the duplex links. However, the fluctuation of the latency is relatively higher for the VoWLAN, which is accurate to real-world scenarios.

UDP - Jitter

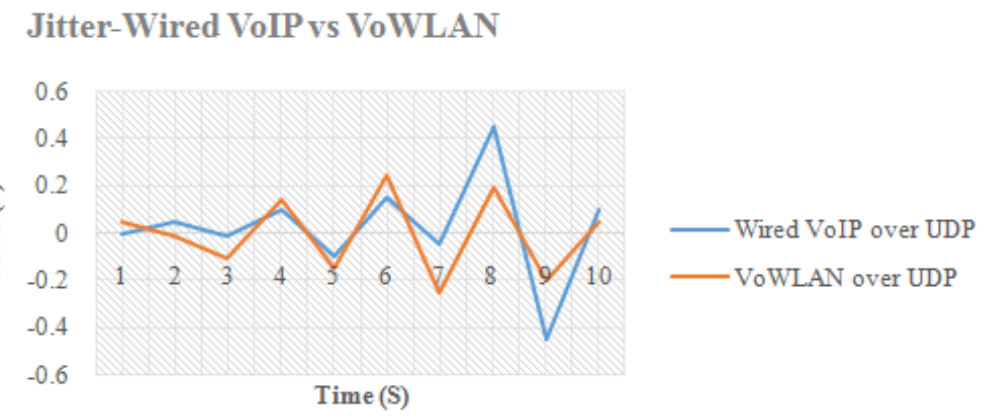

Figure 6: Jitter - Wired VoIP VS VoWLAN

As mentioned in the latency test, the jitter is higher for the wireless network than for the wired. This is also theoretically correct, as wireless signals are prone to interference and signal instability.

\section{TCP - Throughput}

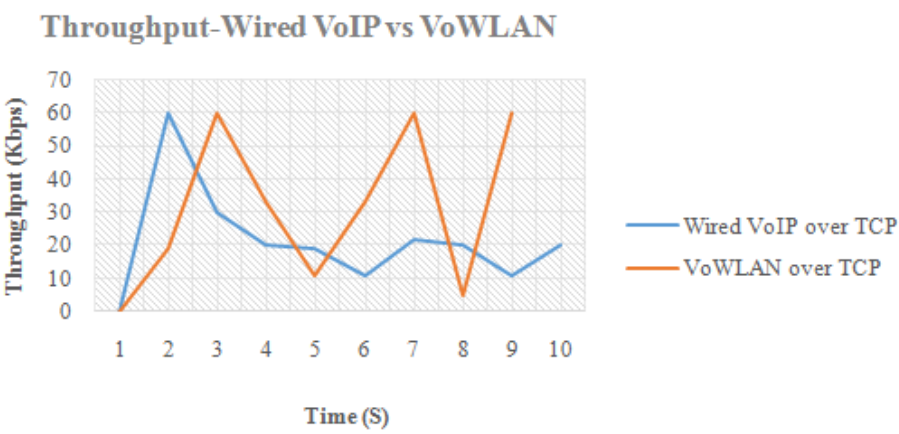

Figure7: Throughput - Wired VoIP VS VoWLAN

The throughput under the TCP protocol remains the same for the wired VoIP network, as the background traffic caps the transmission speed, and the Drop-Tail queuing method becomes a race condition between VoIP and background traffic. However, the wireless network benefits from the error detection of the TCP protocol, which in itself attributes for some of the extra throughput.

\section{TCP - Latency}

\section{Latency-Wired VoIPvs VoWLAN}

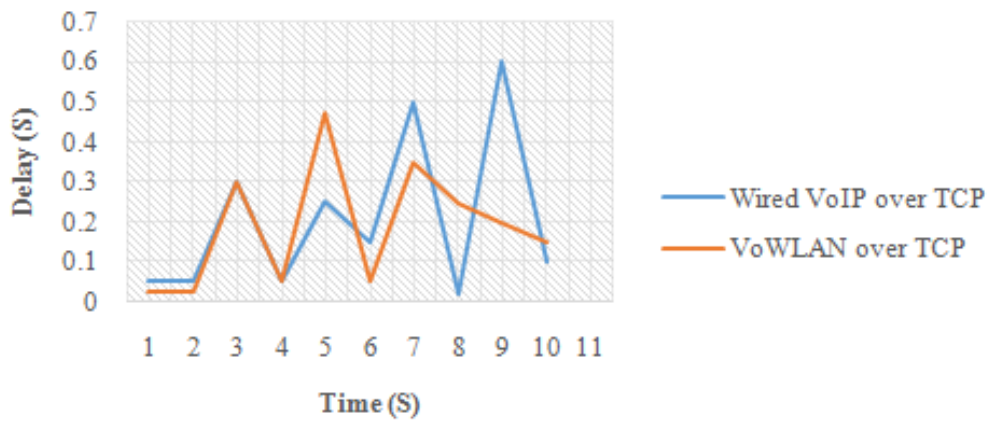

Figure8: Latency - Wired VoIP VS VoWLAN 
Similar to the UDP protocol tests, the TCP results in higher overall latency, but displays the same characteristics for the VoIP latency in the presence of background traffic. The TCP protocol gives a slightly higher latency due to its error detection functionalities. This also results in higher jitter for periods when errors occur within the traffic.

\section{TCP - Jitter}

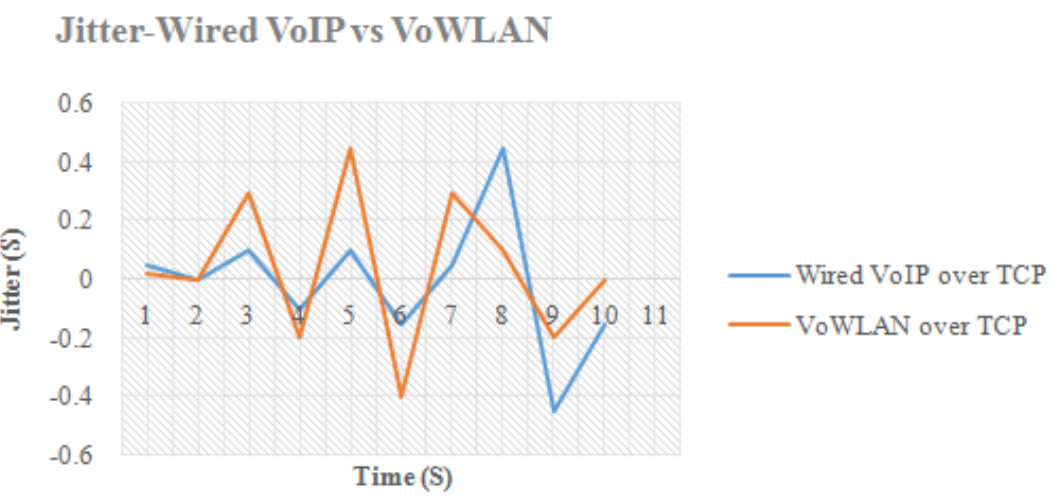

Figure9: Jitter - Wired VoIP VS VoWLAN

The jitter is high in VoWLAN than Wired VoIP but they are higher in TCP as compared to UDP, this is due to the interference from the background traffic that compete with the voice signal as well as error detection.

The following tables summarizes the results of the simulation. The average values can be used to compare the performances between wired and wireless networks for both UDP and TCP protocols.

Table 3 - Average Simulation Results of UDP

\begin{tabular}{|l|l|l|}
\hline UDP & Wired VoIP & VoWLAN \\
\hline Throughput(kbps) & 21.5 & 23.5 \\
\hline Latency(s) & 0.118 & 0.111 \\
\hline Jitter (s) & 0.024 & 0.03 \\
\hline
\end{tabular}

Table 4 - Average Simulation Results of TCP

\begin{tabular}{|l|l|l|}
\hline TCP & Wired VoIP & VoWLAN \\
\hline Throughput(kbps) & 21.3 & 28.752 \\
\hline Latency(s) & 0.207 & 0.1325 \\
\hline Jitter(s) & 0.015 & 0.075 \\
\hline
\end{tabular}

\section{Conclusion}

The purpose of the project is to evaluate the performance difference between wired and wireless VoIP networks. The scenarios are created to include a variety of real-world cases, such as for both UDP and TCP protocols, and with the addition of background traffic [6]. The results are conclusive for the cases of throughput, packet loss, latency, and jitter. The wireless nodes are affected by its proximity to the Access Point, acting as the receiver. In close proximity, the wireless nodes will perform better than the wired network, which had a pre-set delay of $5 \mathrm{~ms}$ for each of the duplex links.

\section{References}

[1]. Wikipedia. "Voice Over IP,” Available: https://en.wikipedia.org/wiki/Voice_over_IP [Accessed: January 27, 2016]

[2]. Wikibooks. "TCP and UDP Protocols," Available:

https://en.wikibooks.org/wiki/Communication_Networks/TCP_and_UDP_Protocols.html [Accessed: January 27, 2016]

[3]. NS3 doxygen "NS3 Documentation," https://www.nsnam.org/doxygen/ [Äccesses: January 27, 2016]

[4]. CISCO, "Voice Over IP - Per Call Bandwidth Consumption," Available: http://www.cisco.com/c/en/us/support/docs/voice/voicequality/7934-bwidth-consume.html [Accessed: January 27, 2016]

[5]. Wikipedia. "Packet delay variation," Available: http://en.wikipedia.org/wiki/Packet_delay_variation [Accessed: January 27, 2016]

[6]. Hole, D.P. Tobagi, F.A. "Capacity of an IEEE 802.11b Wireless Lan supporting VoIP," Communications, 2004 IEEE International Conference on, vol.1, no., pp. 196-201 